\title{
Prolapsus stomial : sucrer avant réduction !
}

\section{Stoma Prolapse: Add Some Sugar Before Reduction!}

\section{T. Schmutz $\cdot$ P. Stengel $\cdot$ V. Ribordy}

Reçu le 11 février 2019; accepté le 26 février 2019

(C) SFMU et Lavoisier SAS 2019
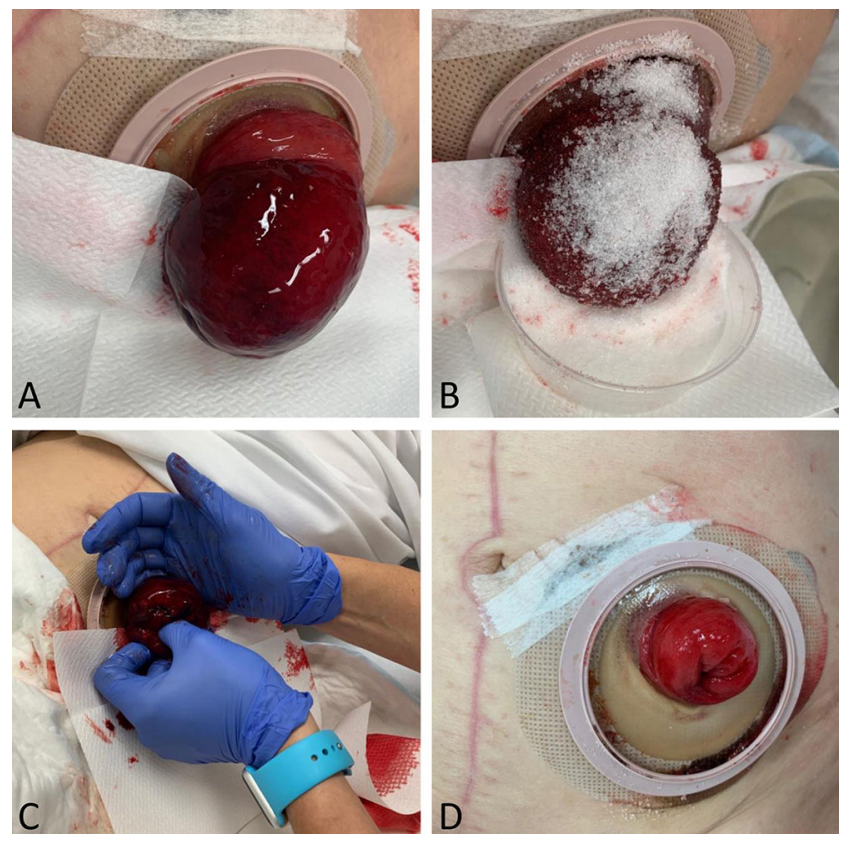

Fig. 1 A. Prolapsus stomial œdématié. B. Application de sucre de table sur le segment prolabé. C. Réduction manuelle après un effet osmotique de 30 minutes. D. Prolapsus réduit avec un léger œdème muqueux persistant
Le prolapsus stomial est la complication la plus fréquente après la confection d'une stomie. Il peut être muqueux (simple éversion de la muqueuse) ou total avec déroulement de l'intégralité de la paroi digestive. L'œdème consécutif à la stase veineuse qui s'installe après l'éversion de l'anse digestive à travers la stomie peut rapidement rendre impossible la réduction manuelle. Il risque de conduire à l'étranglement $\mathrm{du}$ prolapsus. Il est alors non réductible, douloureux et entraîne la nécrose de l'anse intestinale qui va devenir cyanosée puis noire. C'est une urgence chirurgicale. En présence d'un prolapsus simple, même très œdématié, l'application de sucre de table (ou de compresses imbibées de mannitol) sur le segment prolabé pendant une trentaine de minutes (Fig. 1) réduit l'œdème par effet osmotique et provoque ou facilite la réduction [1]. Un œdème muqueux peut persister après la manœuvre (Fig. 1). Ici, l'évolution a été favorable, évitant une chirurgie en urgence. La même technique peut être utilisée pour réduire un prolapsus rectal [2]. Des pansements sucrés sont aussi décrits pour diminuer l'œdème des plaies.

\section{Références}

1. Brandt ARML, Schouten O (2011) Sugar to reduce a prolapsed ileostomy. N Engl J Med 364:1855

2. Myers JO, Rothenberger DA (1991) Sugar in the reduction of incarcerated prolapsed bowel. Dis Colon Rectum 4:416-8 